\title{
A Rare Dissociation at the Neck-Stem Interface
}

\author{
A. Kouzelis ${ }^{\dagger}$, C.S. Georgiou ${ }^{\dagger}$, and P.Megas ${ }^{\dagger}$
}

The authors report a case of dissociation at the neckstem interface without hip dislocation that occurred during walking, and discuss strategies to avoid and treat this complication of THA.

\section{Introduction}

Modular femoral components have the advantages of:

- Reducing the need to stock numerous stem and head sizes

- Allowing the final choice of neck length and head size to be made after stem implantation

With moduar femoral components, neck orientation can also be changed after implantation, which is a well-known cause of early dislocation. The incidence of postoperative dislocation of modular total hip arthroplasty (THA) varies from $0.5 \%$ to $4 \%$ [1].

Dissociation at the neck-stem interface is rare. To the best of our knowledge, only three case reports have been published [2-4], but they pertained to dissociation at the neck-head interface.

We report a case of dissociation at the neck-stem interface without hip dislocation that occurred during walking, and we discuss the causes of dissociation as well as strategies to avoid and treat this complication.

\section{Case Presentation and Management}

A 72-year-old man had undergone a right THA in 1996. Revision THA was performed in our institution in 2005 due to aseptic loosening of both components.

After intraoperative extraction of the acetabular shell, we determined that a jumbo acetabular com- ponent (Procotyle, Wright) was needed to manage the serious acetabular bone loss that was discovered. Allograft augmentation of the acetabulum was also used to repair the defect.

The acetabular shell was $60 \times 68 \mathrm{~mm}$ in outer diameter; additional fixation was achieved with three cancellous screws. The polyethylene liner was group $2,15^{\circ}, 28 \mathrm{~mm}$ in inner diameter.

For the femo-

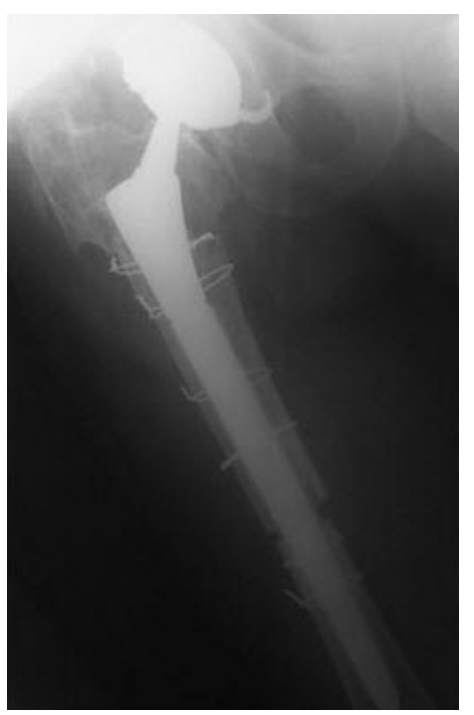

Figure 1. Radiograph after the first operation reveals good relationship of the total hip prosthesis with acetabular and femoral bones. ral component, which was fully porous-coated and therefore distally fixed, we used a modular stem (Profemur-R, Wright). The open-book technique was used to extract it, and a transverse osteotomy just under the tip was also made, which we use in such cases to avoid distal extension of the osteotomy (openbook technique) and to preserve good bone stock for the distally fixed stem.

Postoperative radiographs revealed adequate positioning of the THA components (Figure 1). The usual protocol for THA postoperative treatment was used, and patient mobilization began on the second postoperative day. The patient was discharged on the eighth postoperative day, fully mobilized (partial weight-bearing) and without residual problems. 
The patient gave informed consent to publish this case.

\section{Postoperative Course}

The usual clinical and radiographic follow-up during the first and third months was normal. The patient was satisfied with the result of the operation and was mobilized with two canes, according to the instructions of the surgeon.

One month later (4 months postoperative), the patient arrived at our emergency department unable to walk and with pain in the revised hip. At clinical presentation, he reported an incident of sudden pain and then falling during normal walking and with no extreme hip movement or rotation. Radiographs revealed dissociation of the modular stem at the femoral neck-stem interface without dislocation of the head (Figure 2).

Immediate revision surgery was performed to reaffix the neck to the main body of the prosthesis. During the operation, stability testing of the acetabular shell revealed adequate fixation of the prosthesis. A new modular interchangeable neck system was implanted; however, as this type of stem also has a modular proximal component, we decided to change it to prevent further complication at the proximal component-stem junction.

All intraoperative stability and orientation tests were normal. Postoperative radiographs were normal (Figure 3).

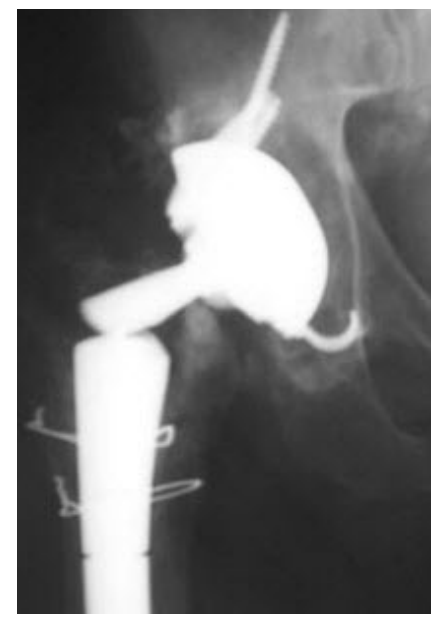

Figure 2. Radiograph shows dissociation of the femoral neck-stem interface.

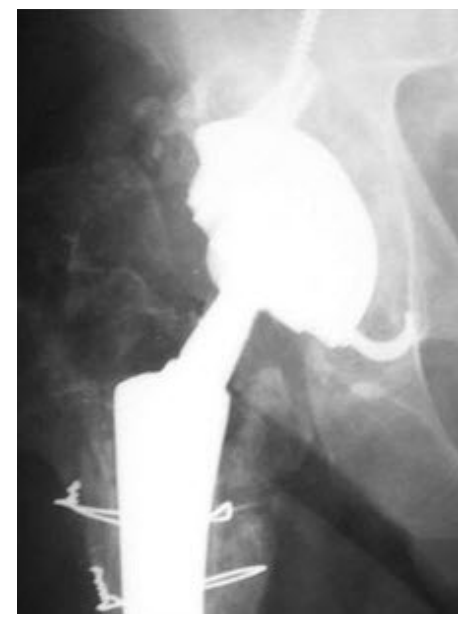

Figure 3. Radiograph after the second operation reveals reimpacted new femoral neck-stem component. Notice the absence of ectopic bone from the lesser trochanter area.

\section{Discussion}

The use of modular components greatly increases flexibility during THA, but also introduces the risk of failures at the interfaces and possible intraoperative errors in matching. Dislocation is a potential problem after THA $[1,5,6]$, and dissociation of modular components after dislocation is unique to modular systems.

Dissociation can occur during closed reduction of dislocation at two different interface levels: the fixed acetabular shell-polyethylene liner interface [2,713], and the femoral head-neck interface [2-4]. In our case, dissociation occurred at the femoral neckstem interface, with no previous traumatic incidence. To the best of our knowledge, no such case concerning this type of prostheses had previously been reported.

The manner in which this incident occurred reveals inadequate modular component fixation or a repetitive force that provoked micromovement of the modular interface that finally led to component dissociation. Potential causes of dissociation during normal walking are as follows:

Inadequate orientation of femoral neck resulting in stress forces at the stem-neck interface. In our case, orientation of the femoral and acetabular components cannot be reliably evaluated due to the absence of a computed tomography (CT) scan of the indexed hip.

Excessive telescopic movements, which finally led to dissociation by creating negative pressure in the acetabular area. Computer-assisted measurement of distal stem migration showed a subsidence of 3.6 $\mathrm{mm}$ at 3 months, which is considered excessive for this short postoperative period, though it is expected for this type of revision stem and transfemoral approach [14] (Figure 4).

Such an early stem subsidence and subsequent leg shortening can result in loss of intraoperative soft tissue tension and, eventually, in hip-joint instability.

Impingement of the femoral neck at the acetabular shell or at osteophytes in the area, causing mechanical stresses at the finally dissociated interface. As mentioned above, component-to-component impingement cannot be confirmed in our case. However, we consider bony impingement to be more important for this patient. Arc length between the tip of the greater trochanter and the ilium (GT arc) has been shown to correlate with free hip flexion and abduction before impingement [15]. In this case, mini- 

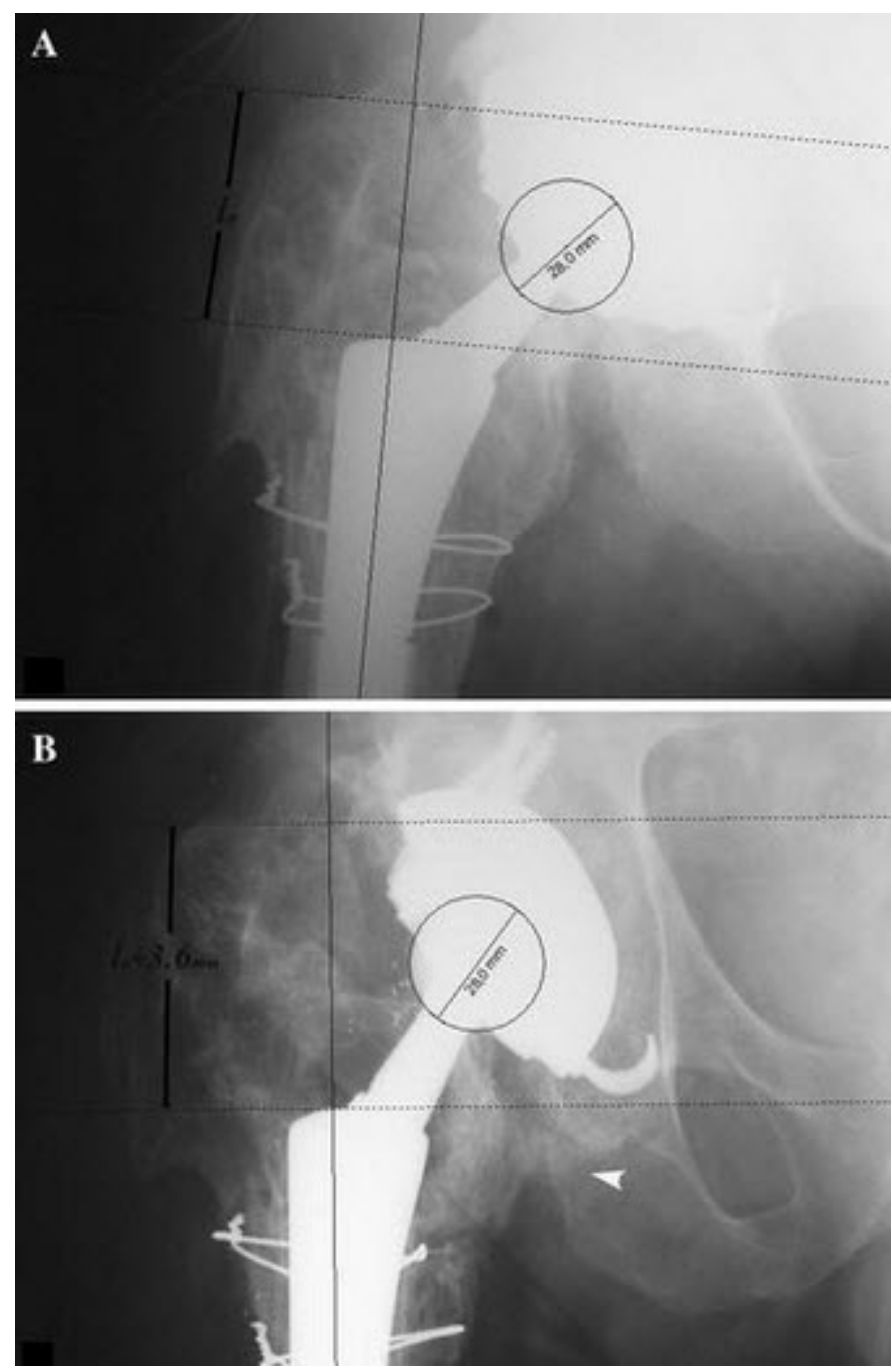

Figure 4. Stem subsidence was measured by processing immediate postoperative (a) and 3-month (b) follow-up anteroposterior radiographs via Roman v1.7 software (Roman free to share software version V1.70; Robert Jones and Agnes Hunt Orthopaedic Hospital, Oswestry, UK; http://www.Keele.ac.uk/depts./ rjah/), as a change in the vertical distance from the proximal tip of the greater trochanter to the shoulder of the stem. Ectopic bone formation at the lesser trochanter area (white arrowhead) is noted 3 months postoperatively (b).

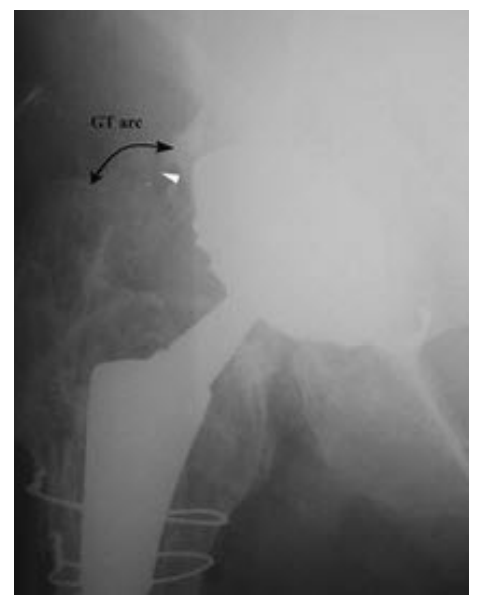

Figure 5. Minimal greater trochan ter $(G T)$ arc length and high position of the tip of the GT in relation to the head center predicts early impingement of the GT to the ilium. A bone spur (osteophyte) at the tip of the GT (white arrowhead) may further limit impingement-free range of motion. mal arc length and the high position of the tip of the greater trochanter in relation to the head center predicts early bony impingement (greater trochanter to ilium) (Figure 5).

In a computer model, it has been shown that once bony impingement becomes the restricting factor, further changes in implant design and orientation may not improve range of motion (ROM) [15]. Furthermore, in a cadaver study of hip dislocation, osseous impingement was likely to occur between the greater trochanter and the iliac wing before component impingement [16]. Similarly, bony impingement preceded component impingement in about $44 \%$ of all conditions tested in a three-dimensional computer model with varying orientations of the femoral and acetabular components [17].

Ectopic bone formation causing abnormal movement of the joint. Heterotopic ossification can cause hip-joint instability when the periarticular bone mass limits femoral excursion or contributes to impingement [18]. However, to our knowledge only in two cases was hip dislocation directly attributed to heterotopic ossification [19].

Modular titanium alloy neck adapters, such as the one used in our case, can fail due to surface micromotions, according to recent retrieval examinations and biomechanical simulation [20]. Whether this movement leads, apart from fatigue fracture, to neck dissociation is unclear. Nevertheless, in large case series with similar neck adapters applied, no case of dissociation was reported [21].

In our case, a jumbo cup was used due to extensive bone loss to ensure stable primary fixation. Three cancellous screws were also placed for the same reason. Regarding the femur, the main goal was successful diaphyseal fixation of the stem; therefore, a long, fully porous-coated, trapezoid-shaped stem was used.

For the modular neck, a straight $0^{\circ}$ long neck was selected, allowing fine positioning of the stem in relation to the cup. Although unnecessary [22], three medium hammer blows were applied to fix the neckstem coupling.

Intraoperatively, during the second revision, a large amount of ectopic bone was found in the lesser trochanter area, which is a possible cause of stem impingement and, in particular, the neck-stem interface, which may lead to dissociation due to repetitive stresses and micromovement in the area. The ectopic bone was removed, and intraoperative mobilization revealed free movement of the hip joint in all pos- 
sible directions.

Modular components give the surgeon an intraoperative advantage but also increase the potential for component mismatch and mechanical failure. Dissociation is a rare but possible cause of failure.

To prevent this complication, the femoral neck component should be impacted firmly onto the tapered stem base during the operation. Finally, free movement of the joint is essential to prevent abnormal stresses at the interfaces of the modular components.

\section{Source}

Kouzelis A, Georgiou CS, Megas P. Dissociation of modular total hip arthroplasty at the neck-stem interface without dislocation. Journal of Orthopaedics and TraumatologyOfficial Journal of the Italian Society of Orthopaedics and Traumatology (C) The Author(s) 201110.1007/s10195-011-0172-9. http:// link.springer.com/article/10.1007/s10195-011-01729/fulltext.html

This article is distributed under the terms of the Creative Commons Attribution License which permits any use, distribution and reproduction in any medium, provided the original author(s) and source are credited.

\section{References}

1. Carlsson AS, Gentz CF (1977) Postoperative dislocation in the Charnley and Brunswik total hip arthroplasty. Clin Orthop 125:177-182

2. Star MJ, Colwell CW Jr, Donaldson WF III, Walker RH (1992) Dissociation of modular hip arthroplasty components after dislocation: a report of three cases at differing dissociation levels. Clin Orthop 278:111-115

3. Chu CM, Wang SJ, Lin LC (2001) Dissociation of modular total hip arthroplasty at the femoral head-neck interface after loosening of the acetabular shell following hip dislocation. J Arthroplast 16(6):806-809

4. Woolson ST, Pottorff GT (1990) Disassembly of a modular femoral prosthesis after dislocation of the femoral component: a case report. J Bone Joint Surg Am 72(4):624-625

5. Gill TJ, Sledge JB, Müller ME (1998) The Bürch-Schneider anti-protrusio cage in revision total hip arthroplasty: indications, principles and long-term results. J Bone Joint Surg Br 80(6):946-953
6. Lewinnek GE, Lewis JL, Tarr R, Compere CL, Zimmerman JR (1978) Dislocations after total hip-replacement arthroplasties. J Bone Joint Surg Am 60(2):217-220

7. Cameron HU (1993) Dissociation of a polyethylene liner from an acetabular cup. Orthop Rev 22(10):1160-1161

8. Wilson AJ, Monsees B, Blair VP III (1988) Acetabular cup dislocation: a new complication of total joint arthroplasty. AJR 151(1):133-134

9. Kitziger KJ, DeLee JC, Evans JA (1990) Disassembly of a modular acetabular component of a total hip-replacement arthroplasty. A case report. J Bone Joint Surg Am 72(4):621-623

10. Ries MD, Collis DK, Lynch F (1992) Separation of the polyethylene liner from acetabular cup metal backing. A report of three cases. Clin Orthop Relat Res 282:164-169

11. Bueche MJ, Herzenberg JE, Stubbs BT (1989) Dissociation of a metalbacked polyethylene acetabular component. A case report. J Arthroplast 4(1):39-41

12. Ferenz CC (1988) Polyethylene insert dislocation in a screw-in acetabular cup. A case report. J Arthroplast 3(3):201-204

13. O'Brien RF, Chess D (1992) Late disassembly of a modular acetabular component. A case report. J Arthroplast 7 Suppl:453-455

14. Artiaco S, Boggio F, Titolo P, Zoccola K, Bianchi P, Bellomo F (2011) Clinical experience in femoral revision with the modular Profemur R stem. Hip Int 21(1):39-42

15. Bunn A, Colwell CW Jr, D’Lima DD (2011) Bony impingement limits design-related increases in hip range of motion. Clin Orthop Relat Res. (in press) [Epub ahead of print]

16. Bartz RL, Nobel PC, Kadakia NR, Tullos HS (2000) The effect of femoral component head size on posterior dislocation of the artificial hip joint. J Bone Joint Surg Am 82(9):1300-1307

17. Kessler O, Patil S, Wirth S, Mayr E, Colwell CW Jr, D’Lima DD (2008) Bony impingement affects range of motion after total hip arthroplasty: a subject-specific approach. J Orthop Res 26(4):443-452

18. Iorio R, Healy WL (2002) Heterotopic ossification after hip and knee arthroplasty: risk factors, prevention, and treatment. J Am Acad Orthop Surg 10(6):409-416

19. Cobb TK, Berry DJ, Wallrichs SL, Ilstrup DM, Morrey BF (1999) Functional outcome of excision of heterotopic ossification after total hip arthroplasty. Clin Orthop Relat Res 361:131-139

20. Grupp TM, Weik T, Bloemer W, Knaebel HP (2010) Modular titanium alloy neck adapter failures in hip replacement - failure mode analysis and influence of implant material. BMC Musculoskelet Disord 11:3

21. Toni A, Sudanese A, Paderni S, Guerra E, Bianchi G, Antonietti B, Giunti A (2001) Cementless hip arthroplasty with a modular neck. Chir Organi Mov 86(2):73-85

22. Pallini F, Cristofolini L, Traina F, Toni A (2007) Modular hip stems: determination of disassembly force of a neck-stem coupling. Artif Organs 31(2):166-170 